\title{
LA COMPETITIVIDAD GLOBAL
}

\author{
GLOBAL COMPETITIVENESS
}

Recepción: 25/09/2017

Aceptación: 17/11/2017

Publicación: 25/12/2017

\section{Resumen}

En este foro mundial se trata de medir la competitividad, capacidad de 137 países que buscan mantener un rendimiento en sus inversiones que es un factor clave para crecimiento económico de las naciones. Este foro identifica las fortalezas y las debilidades; y principalmente lo que busca es que se den cambios óptimos en cada una de sus desventajas y debilidades.

El Foro Mundial facilita su cálculo agrupando en 12 pilares, donde se puede analizar cada ventaja y desventaja de cada uno.

Palabras clave: factores, rendimiento, institucionales, indicadores, sofisticación.

\section{Abstract}

This global forum is to measure competitiveness, capacity from 137 countries seeking to maintain a return on their investment which is a key factor for economic growth of nations. The forum identifies strengths and weaknesses, and you are looking primarily to give optimal changes in each of its drawbacks and weaknesses.

The Global Forum gathering facilitates calculation on 12 pillars, where you can examine each advantage anddisadvantage of each.

Keywords: factors, performance, institutional, indicators, sophistication.

\section{La Competitividad}

La competitividad según el Foro Económico Mundial (2017) es un "conjunto de instituciones, políticas y factores que determinan el nivel de productividad de un país"; así fue como definió esta institución que se dedica a medir las diferencias institucionales que existen en los países, sus observaciones radican en el mercado, la libertad, propiedades privadas, entre otros indicadores.

A otro nivel como el microeconómico se puede entender que la competitividad es cuando las empresas miden su capacidad para competir en el mercado, logrando conseguir sus beneficios económicos y expandirse; en lo macro se entiende por competitividad al nivel de capacidad que tiene un país por competir a niveles de mercado abierto, producción de bienes y servicios por otras economías que hacen crecer la renta nacional.

Así mismo, la competitividad se la puede entender como la capacidad de una organización de mantener sistemáticamente ventajas comparativas que le permitan alcanzar, sostener y mejorar una determinada posición en el entorno socioeconómico. La ventaja comparativa de una empresa estaría en su habilidad, recursos, conocimientos, atributos, etc., características que carecen sus competidores, esto le permite a la empresa obtener rendimientos superiores a los de su competencia.

En cuanto al sector empresarial, la competitividad se puede dar en precios, por ejemplo: 
una empresa puede ofrecer sus productos o servicios a un precio que le permita cubrir los costos de producción y su rendimiento sobre el capital que ha sido invertido.

Para muchos la "competitividad" es solo un concepto relativo que muestra los sistemas de empresas, sectores, países, se considera como concepto de desarrollo, que está sujeto a muchos cambios, mediciones e interpretaciones. Según el Centro de Estudios de Competitividad (2012) en términos generales la competitividad es "la capacidad para competir en los mercados por bienes o servicios"; también este término se utiliza con frecuencia para justificar la implementación de políticas públicas.

Según la Real Academia Española (2017) define a la "competitividad" como la capacidad de competir.

La competitividad según Michael Porter (Profesor de la Universidad de Harvard, 2015) es la "productividad que un país utiliza sus recursos humanos, económicos y naturales", todo a partir de las "fuentes subyacentes" de prosperidad que exista. Según Porter para analizar la "competitividad" se toma en cuenta muchos factores, para esto resulta de mucha importancia las escuelas, carreteras, mercados financieros; estos son uno de los tantos factores que mejoran la competitividad en los países.

El concepto de competitividad está muy estrechamente relacionado con los conceptos de productividad, calidad y proceso:
La productividad es la relación entre las entradas (insumos) y salidas (productos) de un sistema productivo. Con frecuencia la productividad es medida mediante la relación entre las salidas divididas para las entradas. Si se produce más salidas con las mismas entradas se mejora la productividad, también aumentan la productividad si se utilizan menos entradas para producir la misma cantidad de salidas.

La calidad se la puede entender como: aquellas características del producto que llegan a satisfacer las necesidades del consumidor, consiste en productos y procesos que no tengan deficiencias, y es una calificación que resulta de comprar el producto con estándares calificados.

El proceso es una serie de actividades entre sí que convierten insumos en productos, esas actividades agregan valor al producto final.

\section{La Metodología que se Utiliza para Elaborar el Informe Global de Competitividad}

Michael Porter que es uno de pensadores que influyen en la gestión de las empresas; fue el que asesoró a la World Economic Forum (WEF), que es la encargada de elaborar el Informe Mundial de Competitividad de alrededor de 137 países del mundo y con más de 113 variables.

Se han agrupado componentes en tres factores que han determinado la competitividad en 12 pilares:

\section{Figura 1. Factores que Determinan la Competitividad}
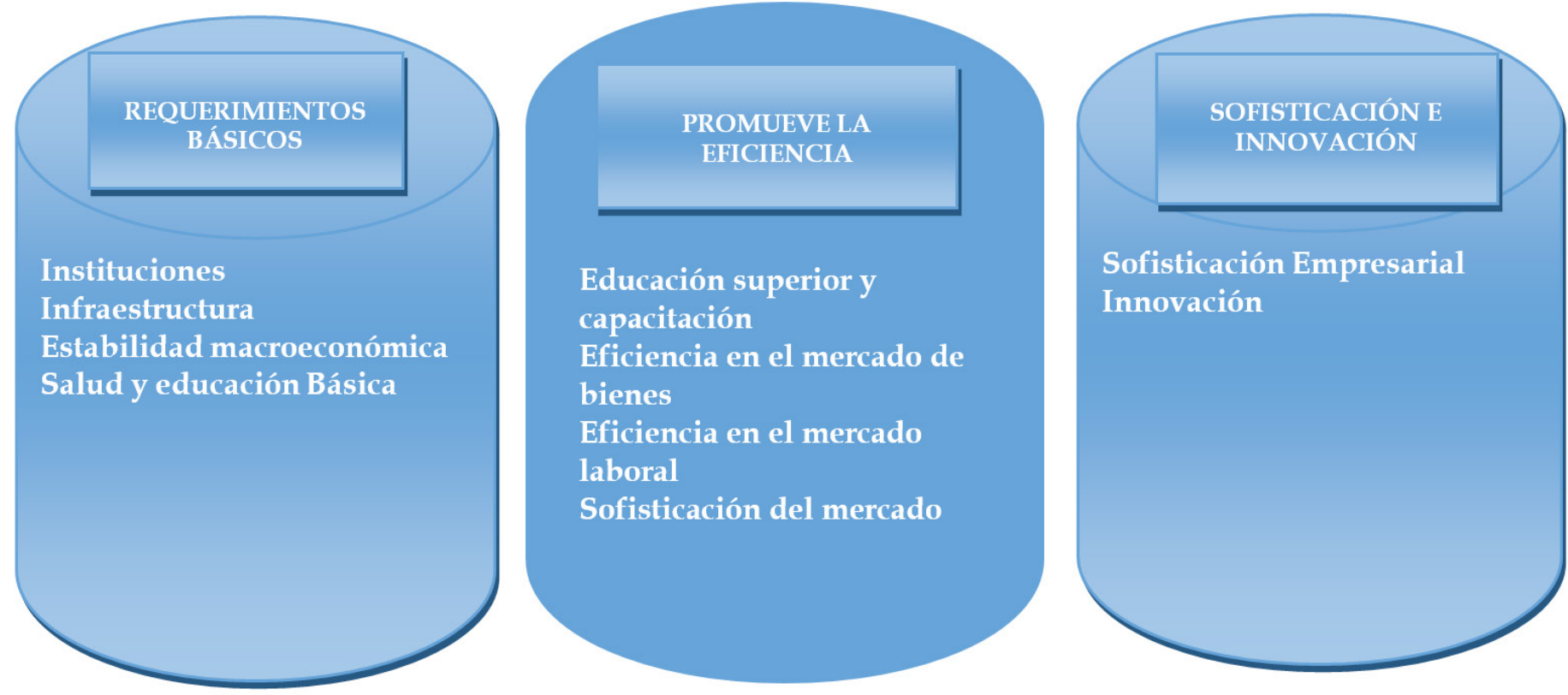


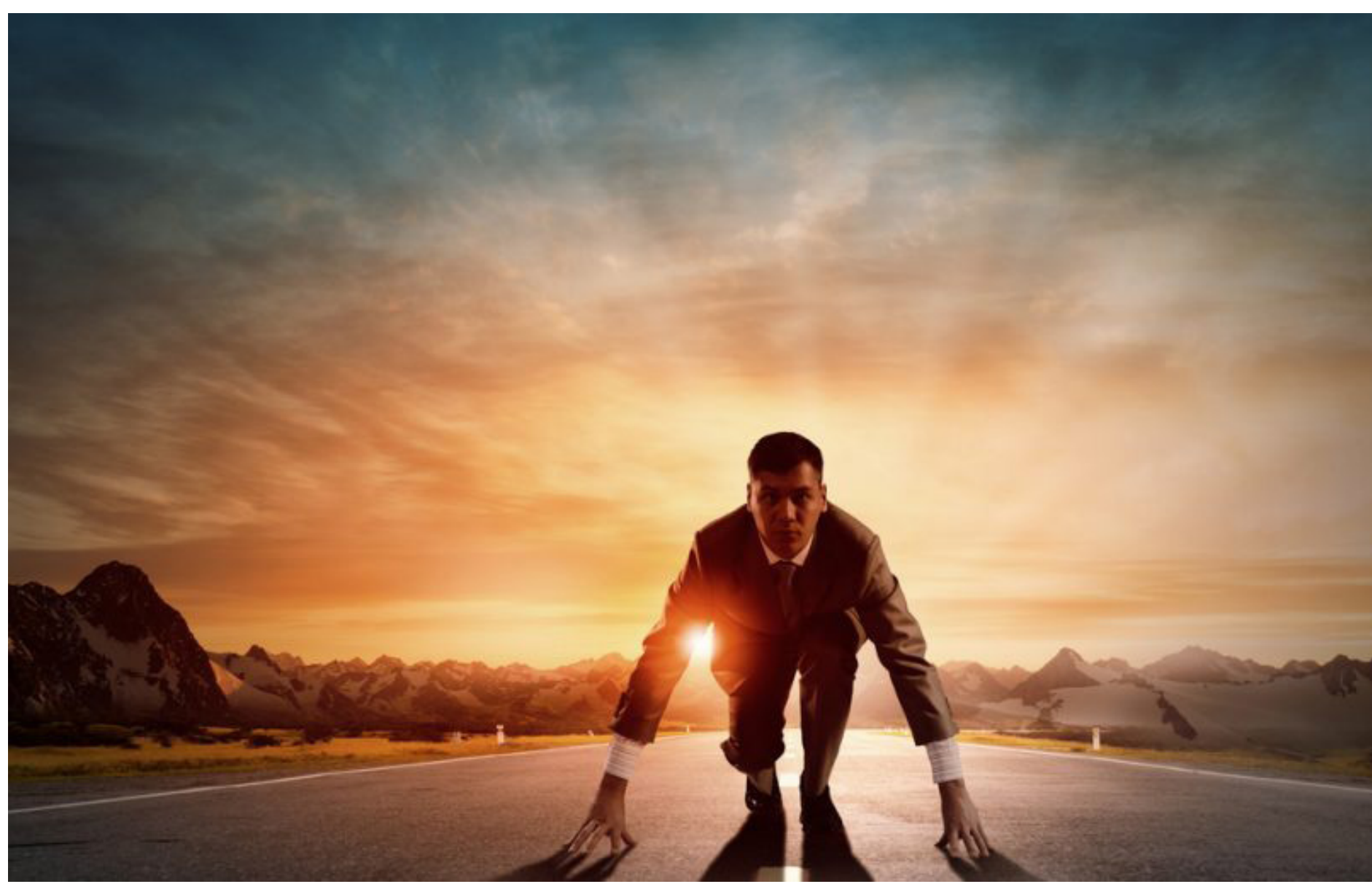

Fuente: Informe de Competitividad Global, Foro Económico Mundial.

\section{El Informe de Competitividad Mundial o Global}

El informe sobre competitividad mundial permite medir la competitividad de los países y a su vez hacer un estudio entre los países más destacados por sus factores que inciden en el crecimiento económico y la competitividad; el informe busca aclarar las diferencias en la evolución del ingreso percápita.

Según el Informe de Competitividad Mundial 2017-20181, publicado por el Foro Económico Mundial, ubica a Suiza por noveno año consecutivo en el primer lugar del ranking de competitividad mundial. Estados Unidos sube un puesto, y en tercer puesto se sitúa ahora Singapur, seguidas de Holanda y Alemania. Estos países están entre los diez primeros puestos, conjuntamente con los Hong Kong, Suecia, Reino Unido en los puestos 6,7 y 8 respectivamente; les siguen Japón (9), y Finlandia (10) completan la clasificación de las diez economías más competitivas. En cambio, en los últimos lugares de competitividad se ubican Chad (135), Mozambique (136), Yemen (137).
Tabla 1. Países más competitivos 2017-2018

\begin{tabular}{|l|c|}
\hline $\begin{array}{c}\text { Los Cinco Países Más Competitivos (Índice de Com- } \\
\text { petitividad Global 2017-2018) }\end{array}$ \\
\hline Países & 1 \\
\hline Suiza & 2 \\
\hline Estados Unidos & 3 \\
\hline Singapur & 4 \\
\hline Holanda & 5 \\
\hline Alemania & \\
\hline
\end{tabular}

Fuente: Informe de Competitividad Global, Foro Económico Mundial.

Suiza con una población de 8.1 millones, es a nivel mundial el país que mejores resultados ha obtenido en todos los aspectos de competitividad, las instituciones científicas de investigación suizas están entre las mejores del mundo y tienen una estrecha colaboración lo académico y lo empresarial permitiendo que los productos y sus procesos estén protegidos por la propiedad intelectual. Otro lado destacable es que las instituciones públicas se encuentran entre las más eficaces y transparente del mundo. El gobierno busca mejorar la confianza empresarial, haciendo que el poder judicial sea independiente. En cuanto al sector 
macroeconómico es considerado como una de las economías más estables en el mundo.

Estados Unidos que ocupa el segundo lugar, lidera en innovación, tecnología. Singapur está en el tercer lugar en competitividad, cuenta con una población de 5.3 millones; sus instituciones públicas y privadas son consideradas como las mejores del mundo. Se encuentra en los primeros lugares en cuanto a la eficiencia de sus bienes y los mercados, y en cuanto al desarrollo del mercado financiero. En la educación se han preocupado por mejorarla cada año y convertirla es su pilar, de esta manera logra que sus habitantes tengan más habilidades en esta economía que cambia cada vez más rápido.

\section{La Competitividad en la Región}

A nivel de Latinoamérica los mejores países ubicados en el ranking de competitividad mundial 2017-2018 son: Chile No 33, Costa Rica $N^{\circ} 47$, Panamá No 50 , México No 51 , Colombia $\mathrm{N}^{\circ} 66$, Perú $\mathrm{N}^{\circ} 72$.

Chile es el país de la región que mejor se encuentra ubicado en el ranking de competitividad mundial, con una población de 17.6 millones de personas ocupa el puesto 33 en competitividad mundial, alcanzó la mayor puntuación a nivel de cobertura de necesidades básicas, estabilidad económica y aspectos institucionales. Su aspecto de economía estable lo convierte en el más competitivo de América Latina; a nivel macroeconómico se encuentra muy bien, tiene niveles bajos de deuda pública, un correcto funcionamiento en las instituciones públicas, estos aspectos importantes hacen que este país tenga una base sólida para seguir manteniéndose con liderazgo en la región, aunque presenta ciertos problemas para hacer negocios: un alto nivel de regulaciones laborales restrictivas, la fuerza de trabajo inadecuada, y una burocracia gubernamental, y tiene una escasa capacidad para innovar.

Costa Rica es el segundo mejor en la región, ha escalado posiciones por tener una percepción positiva en los empresarios, además de existir confianza en los políticos.

Panamá es el tercer país de la región mejor ubicado en competitividad, con una población de 3.6 millones es quien ocupa el puesto 50, se destaca en competencia en mercados y acceso a la tecnología, aprovecha su excelente infraestructura de transporte, especialmente para los puertos, su estabilidad macroeconómica, apertura comercial, servicios financieros, pero tiene problemas para hacer negocios debido a la corrupción, burocracia y delincuencia.

Con una población de alrededor 199.7 millones, Brasil conocida por su comunidad empresarial y se encuentra en el lugar 80; uno de sus principales desafíos es la desconfianza en sus políticos que sigue siendo baja, otra parte no alentadora es que a pesar del esfuerzo para facilitar el espíritu empresarial, todavía en las pequeñas empresas, los procedimientos y el tiempo para iniciar un negocio son más altos de la muestra; además de impuestos que están por las nubes.

Venezuela con una población de 29.7 millones se encuentran en el lugar 127 de competitividad mundial, en este país sigue creciendo la desconfianza en los negocios, su macroeconomía tiene muchos problemas. Otro gran problema es que las normas y regulaciones no permiten que la inversión extranjera directa llegue a este país.

\section{Tabla 2. Países Latinoamericanos mejor ubicados en competitividad 2017-2018}

\begin{tabular}{|l|c|}
\hline $\begin{array}{c}\text { The Global Competitiveness Index 2017-2018 Rankings } \\
\text { Latin American }\end{array}$ \\
\hline \multicolumn{1}{|c|}{ Country/Economy } & Rank \\
\hline Chile & 33 \\
\hline Costa Rica & 47 \\
\hline Panamá & 50 \\
\hline México & 51 \\
\hline Colombia & 66 \\
\hline
\end{tabular}

Fuente: Informe de Competitividad Global, Foro Económico Mundial.

\section{La Competitividad en el Ecuador}

En el Informe Mundial de Competitividad 2017-2018, nuestro país se encuentra en el puesto 97 de un total 137 naciones estudiadas; el año pasado se ubicó en el puesto 91 entre 138. Ese valor sitúa Ecuador en el puesto número 97, es decir que tiene un nivel de competitividad mundial bastante deficiente si lo comparamos con el resto de los 140 países del ranking. Ha empeorado su situación, ya que en 2016 estaba en el puesto 76.

Según Wong (2017) existen otro tipo de indicadores que causaron problemas para los negocios, como la inestabilidad política y las regulaciones laborales, como los indicadores de institucionalidad, sofisticación de negocios y educación superior (las variables que contribuyen a la caída son: baja matricula en educación secundaria y en educación superior, y la calidad de las escuelas de negocios). Uno de los factores peor posicionado es el mercado laboral, ya que existe rigidez en la contratación.

Los principales problemas para hacer negocios en Ecuador según el Informe de Competitividad Global 2017-2018 resalta: perjudican la manera de hacer negocios, las principales trabas radican en la corrupción, inestabilidad política, regulaciones laborales restrictivas, el crimen y robos y burocracia ineficiente.

En la siguiente tabla mostramos la evolución de la posición de Ecuador en el Índice de Competitividad Global. 
Tabla 3. Ecuador - Índice de Competitividad Global

\begin{tabular}{|c|c|c|}
\hline Año & $\begin{array}{c}\text { Ranking de } \\
\text { Competitividad }\end{array}$ & $\begin{array}{c}\text { Índice de } \\
\text { Competitividad }\end{array}$ \\
\hline 2017 & $97^{\circ}$ & 3,91 \\
\hline 2016 & $76^{\circ}$ & 4,07 \\
\hline 2014 & $71^{\circ}$ & 4,18 \\
\hline 2013 & $86^{\circ}$ & 3,94 \\
\hline 2012 & $101^{\circ}$ & 3,82 \\
\hline 2011 & $105^{\circ}$ & 3,65 \\
\hline 2010 & $105^{\circ}$ & 3,56 \\
\hline 2009 & $104^{\circ}$ & 3,58 \\
\hline 2008 & $103^{\circ}$ & 3,57 \\
\hline 2007 & $94^{\circ}$ & 3,62 \\
\hline
\end{tabular}

Fuente: https://www.datosmacro.com/estado/indice-competitividadglobal/ecuador

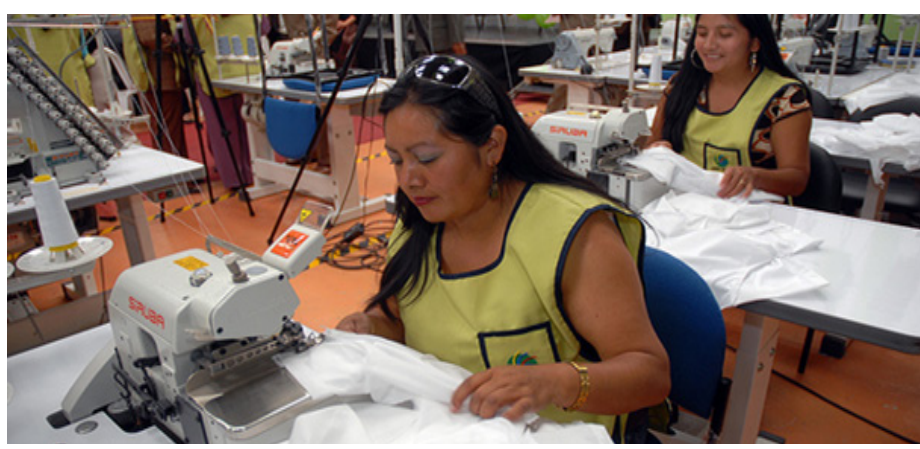

Tabla 4. Factores y pilares de la competitividad en el Ecuador 2017-2018

\begin{tabular}{|l|c|}
\hline \multicolumn{2}{|c|}{$\begin{array}{c}\text { Indice de Competitividad Global 2017-2018 } \\
\text { Tres Factores y Doce Pilares (ECUADOR) }\end{array}$} \\
\hline Indicador & Índice \\
\hline Total & 3.91 \\
\hline I. Requerimientos Básicos & \\
\hline 1. Instituciones & 3.1 \\
\hline 2. Infraestructura & 4.1 \\
\hline 3. Estabilidad Macroeconómica & 4.3 \\
\hline 4. Salud y Educación Primaria & 5.9 \\
\hline II. Factores de Eficiencia & \\
\hline 5. Educación Superior y entrenamiento & 4.3 \\
\hline 6. Eficiencia en los Mercados de Bienes & 3.7 \\
\hline 7. Eficiencia en los Mercados Laborales & 3.4 \\
\hline 8. Sofisticación del Mercado Financiero & 3.3 \\
\hline 9. Preparación Tecnológica & 3.6 \\
\hline 10. Tamaño de Mercado & 3.9 \\
\hline III. Factores de Innovación & \\
\hline 11. Sofisticación de Negocios & 3.6 \\
\hline 12. Innovación & 2.9 \\
\hline
\end{tabular}

Fuente. Informe de Competitividad de Ecuador 2017-2018.

\section{Componente Nacional y Valor Agregado}

De las industrias ecuatorianas la manufacturera es la que ocupa el primer lugar dentro de los sectores que más aporta al Producto Interno Bruto (PIB) con alrededor del $12 \%$. Esto se da al elevado nivel de valor agregado que este sector genera. (Revista Ekos, 2015). Así, dentro de la industria manufacturera la rama que más aporta es la agroindustria con el 30\%; en segundo lugar está el sector metalmecánico con el $11 \%$ y el tercero es la fabricación de sustancias y productos químicos $9,8 \%$ y de donde salen productos y servicios bajo el sello "Hecho en Ecuador".

El aporte de una industria se mide no tanto por el valor de su producción (precio por cantidad), sino por el valor agregado en su producción. El valor agregado se calcula restando el consumo intermedio de la economía del valor bruto de la producción; las compras de bienes y servicios que utilizan las empresas para generar sus productos se denomina consumo intermedio. Todas las industrias tienen en mayor o menor grado un consumo intermedio, que se puede dividir en consumo intermedio importado o consumo intermedio nacional.

El mayor componente nacional de una industria estará determinado por el valor agregado nacional (mientras menor sea el consumo intermedio importado, mayor será el componente nacional de una industria). A nivel de valor agregado nacional la industria que más genera es la fabricación de vidrio, productos refractarios y de cerámica (55\%); fabricación de otros productos químicos (51\%) y elaboración de café (49\%).

En referencia a las industrias que tienen mayor componente nacional son: procesamiento y conservación de camarón (99\%) elaboración y refinación de azúcar (95,47 \%); $\mathrm{y}$ producción de madera $\mathrm{y}$ de productos de madera $(95,41 \%)$. Además, en el caso de productos ensamblados en Ecuador, durante 2014 cambió las regulaciones para subir el componente local mínimo del $5 \%$ al $20 \%$ en el caso de motocicletas; del $13-24 \%$ en celulares; $10-22 \%$ en el caso de televisores; y 19-25\% para radios de vehículos. 
Tabla 5. Top 10 del Valor Agregado y Componente Nacional

\begin{tabular}{|l|c|}
\hline TOP 10: VALOR AGREGADO \\
\hline $\begin{array}{l}\text { Fabricación de vidrio, productos } \\
\text { refractarios y de cerámica }\end{array}$ & $55,41 \%$ \\
\hline $\begin{array}{l}\text { Fabricación de otros productos } \\
\text { químicos }\end{array}$ & $51,18 \%$ \\
\hline Elaboración de café & $49,81 \%$ \\
\hline $\begin{array}{l}\text { Fabricación de cemento, artículos } \\
\text { de hormigón y piedra }\end{array}$ & $46,75 \%$ \\
\hline $\begin{array}{l}\text { Fabricación de productos de } \\
\text { caucho }\end{array}$ & $45,15 \%$ \\
\hline $\begin{array}{l}\text { Fabricación de papel y productos } \\
\text { de papel }\end{array}$ & $41,71 \%$ \\
\hline Fabricación de prendas de vestir & $40,85 \%$ \\
\hline $\begin{array}{l}\text { Industrias manufactureras ncp } \\
\text { Procesamiento de pescado y } \\
\text { otros productos acuáticos } \\
\text { elaborados }\end{array}$ & $40,25 \%$ \\
\hline $\begin{array}{l}\text { Fabricación de cuero, productos } \\
\text { de cuero y calzado }\end{array}$ & $39,65 \%$ \\
\hline
\end{tabular}

\section{TOP 10: CI NACIONAL}

Procesamiento y conservación de camarón $99,57 \%$ Elaboración y refinación de azúcar

$95,47 \%$

Producción de madera y de productos de madera $95,41 \%$

Procesamiento de pescado y otros productos acuáticos elaborados

Elaboración de productos de tabaco

Elaboración de fideos y de otros productos farináceos

\begin{tabular}{|l|c|}
\hline Elaboración de productos lácteos & $92,40 \%$ \\
\hline Fabricación de muebles & $91,84 \%$ \\
\hline Elaboración de café & $91,29 \%$ \\
\hline $\begin{array}{l}\text { Procesamiento y conservación } \\
\text { de carne }\end{array}$ & $90,94 \%$ \\
\hline
\end{tabular}

Por ejemplo, con el aumento de los impuestos arancelarios se limitaron las importaciones de vehículos y se fomentó más el ensamblaje en el país, por lo que las restricciones a la compra de bienes importados más los cupos en el sector automotriz impulsaron la producción nacional de vehículos. Las empresas ensambladoras de vehículos en el país reportan una participación del $51,52 \%$ en el mercado hasta el año 2015.
Según una publicación de diario El Telégrafo (2015), en el año 2014 el sector automotriz del país registró 120.060 vehículos nuevos comercializados, según cifras de la Asociación de Empresas Automotrices del Ecuador (Aeade). Las importaciones generaron 58.205 unidades vendidas, mientras que el ensamblaje local produjo 61.855 automotores comercializados, lo que evidencia un claro ascenso de este último sector. De acuerdo a esos indicadores, el ensamblaje local va ganando terreno. En 2013 el pastel se repartía en $48,77 \%$ correspondiente al ensamblaje y un $51,23 \%$ para las importaciones.

Para 2014, las cifras dieron un giro importante: 51,52\% ensamblaje, y 48,48\% importaciones. La producción de las ensambladoras nacionales (Aymesa, Maresa y Omnibus BB) ascendió al 52\% (61.855 unidades) del consumo local, mientras que en 2013 atendieron al 49\%. El 45,7\% de la producción corresponde a automóviles, $31 \%$ a camionetas, $23 \%$ al ensamblaje de SUV (todo terreno) y $0,3 \%$ a VAN, señala la Aeade en su anuario 2014. En 2013 se importaron 37.654 vehículos nuevos y en 2014 la cifra llegó a 34.337, según datos del Servicio Nacional de Aduana del Ecuador (Senae).

Según el INEC, en el censo económico 2010 indica que en el sector automotriz existen 90.012 personas ocupadas ( $83 \%$ son hombres y $17 \%$ mujeres). En los establecimientos de comercio se encuentran ocupadas 84.155 personas, en manufactura 5.194 y en servicios 663 . Existen 29.068 establecimientos económicos dedicados a actividades de comercio automotriz. El $70 \%$ realiza mantenimiento y reparación de vehículos automotores, el 30\% restante se dedica a la venta de partes, piezas y accesorios de vehículos automotores; venta al por menor de combustibles y venta de vehículos.

\section{Conclusiones}

El aumento de la competitividad en los países implica que sus economías cumplan con ciertas características que le permitan ser más productivas y eficientes. No cabe duda que el aumento de la competitividad en las economías implica un mayor crecimiento de ellas que trae consigo mayor inversión, producción, ingreso e innovación.

En el informe mundial de competitividad 2017-2018, Suiza se mantiene por muchos años en el primer lugar en competitividad debido a la seriedad y eficiencia en el manejo de sus instituciones, la calidad de sus centros de educación y de investigación, la confianza generada en las inversiones del sector empresarial conjuntamente con el sector público.

Mientras Ecuador sigue retrocediendo en el desempeño de la competitividad global 2017-2018, se encuentra en el puesto 97 de un total 137 naciones estudiadas. Dentro de esos factores que han provocado este retroceso en la 
competitividad global se puede señalar: la manera de hacer negocios, la corrupción, inestabilidad política, la contratación laboral, burocracia ineficiente, entre otras.

\section{Listado de Referencias}

Abdel, G. \& Romo, M. D. (2005). Sobre el concepto de competitividad. Revista Comercio Exterior.

Álvarez, C. (2009). Innovación, competitividad y nuevos modelos de negocio. Cuadernos de investigación EPG. Perú

Barandearán, J. (2011). A propósito del emprendimiento ecuatoriano. Humane - Escuela de Negocios. Recuperado Agosto 5, 2012, de http://www. humane.edu.ec/component/content/article/9-noticias/41-a-proposito-delemprendimiento-ecuatoriano.html

Barney, J. (1991). Firm Resources and Sustained Competitive Advantage. Journal of Management

Berumen, S (2006). Una aproximación a los indicadores de la competitividad local y factores de la producción. Cuadernos de administración. Universidad Javeriana

Bosma, N. (Agosto 9, 2012). GEM Consortium. GEM Global Entrepreneurship Monitor. Recuperado Agosto 31, 2012, de http:// gemconsortium.org/docs/download/2412

Cámara de Comercio de Guayaquil (2018), Boletín Económico, Informe de Competitividad Mundial 2017, www.lacamara.org; 2018

Credit Suisse, \& Schwab Foundation for Social Entrepreneurship. (Enero, 2012). Research Institute. Credit Suisse. Recuperado Agosto 30, 2012, de https://infocus.creditsuisse.com/data/_product_documents/_shop/336096/ investing_for_impact.pdf

Cho, Dong-Sung \& Moon, Hwy-Chang (2000). From Adam Smith to Michael Porter. Evolution of Competitiveness Theory. World Scientifc Publishing Co.Pte.

De la Fuente, Osvaldo (2012), Zona Económica-Competitividad, www. zonaeconomica.com/definicion/competitividad; 2012

Fundación Xavier de Salas, \& GEM España (2011). Presentación Proyecto GEM. GEM.

Recuperado Agosto 31, 2012, de http://gem.fundacionxavierdesalas. com/?q=presentacion

\section{INEC (2010), Censo Económico 2010, www.inec.gob.ec}

Kariv, D. (2011). Entrepreneurship: An International Introduction - Dafna Kariv - Google Libros. Google Books. Recuperado Agosto 30, 2012, de http://books.google.com.ec/ooks?id=1 moqmk7NXQCerprintsec=front cover\& dq $=$ Entrepreneurship\&source $=$ bl\&ots $=64 Y 0 z Q I 0 t A \& s i g=s 64$ XjT8GdcjCy0GuSiLqj2oyG8w\&hl=es\&sa=Xઐei=XUJGUOiVEon-8ASjYFQ\&ved=0CEEQ6AEwAw\#v=onepaged $q=$ Entrepreneurshipe $f=$ false

Kelley, D. J., Singer, S., \& Herrington, M. (2011).GEM 2011 Global Report. GEM Global Entrepreneurship Monitor. Recuperado Agosto 30, 2012, de http://www.gemconsortium.org/docs/download/2409

Krugman, P. (1997). El internacionalismo moderno, la economía internacional y las mentiras de la competitividad. Barcelona

Lassio, V., Arteaga, M., ¿Caicedo, G. (2010). Global Entrepreneurship Monitor Ecuador 2010. Guayaquil, Ecuador: ESPAE Graduate School of Management.
NextBillion en Español (Abril 29, 2011). NextBillion.net Desarrollo y Negocios Inclusivos.

NextBillion.net Desarrollo y Negocios Inclusivos. Recuperado Agosto 30, 2012, de http://espanol.nextbillion.net/blogpost.aspx?blogid=411

Porter, Michael E. (1999). "La ventaja competitiva de las naciones". En Michael Porter (ed.).

Ser Competitivo. Nuevas aportaciones y conclusiones. Bilbao: Ediciones Deusto. Págs. 163-202

Porter, Michael E. (1990). "The competitive advantage of nations", Harvard Business Review, vol. 68, no. 2, marzo-abril, pp. 73-93.

Ramon, S. (Julio 8, 2009). Panorama del Emprendimiento en Ecuador y la situación de Loja Sandraramon'sWeblog. Sandraramon's Weblog. Recuperado Agosto 31, 2012, de http://sandraramon.wordpress. com/2009/07/08/panorama-del-emprendimiento-en-ecuador-y-lasituacion-de-lojal

Real Academia de la Lengua (RAE). Concepto de competitividad. http:// buscon.rae.es/draeI/SrvltConsulta?TIPO_BUS=3\&LEMA=competencia

Sara Wong, Ph.D. (2017), Informe de Competitividad de Ecuador. Obtenido de http://www.espae.espol.edu.ec/resultados-del-reporte-decompetitividad-global-2017-2018/

Shane, S. (2003). A General Theory Of Entrepreneurship: The Individual-opportunity Nexus - Scott Andrew Shane - Google Libros. Google Books. Recuperado Agosto 30, 2012, de http://books.google.com. oCw\&ved $=0$ CEEQ6AEwAw\#v=onepageઐ $q=$ Entrepreneurship $\% 20$ ise $f=$ false

University of Oxford (2009). What is social entrepreneurship? Saïd Business School, University of Oxford.Recuperado Agosto 5, 2012, de http://www.sbs. ox.ac.uk/centres/skoll/about/Pages/whatisse.aspx

Valda, J. C. (Septiembre 15, 2011). Retos y desafíos de los emprendedores en América Latina. Grandes Pymes. Recuperado Agosto 31, 2012, de http:// jcvalda.wordpress.com/2011/09/15/retos-y-desafios-de-los-emprendedoresen-America-latinal

Wallener, D., \& Allen, J. (Agosto 30, 2012). What Is an Entrepreneur? wiseGEEK: clear answers for common questions. Recuperado Septiembre 1, 2012, de http://www.wisegeek.com/what-is-an-entrepreneur.htm World Economic Forum (2018), Informe Mundial de Competitividad, www. weforum.org, 2018 